\title{
Desafios cotidianos e possibilidades de cuidado com crianças e adolescentes com Transtorno do Espectro Autista (TEA) frente à COVID-19
}

Amanda Dourado Souza Akahosi Fernandes ${ }^{a}$ (D), Marina Speranza ${ }^{a}$ (D), Mayara Soler Ramos Mazak ${ }^{a}$ (D), Danieli Amanda Gasparini ${ }^{a}$ (D), Maria Fernanda Barboza Cid ${ }^{a}$ (D)

${ }^{a}$ Universidade Federal de São Carlos - UFSCar, São Carlos, SP, Brasil.

Como citar: Fernandes, A. D S. A., Speranza, M., Mazak, M. S. R., Gasparini, D. A., Cid, M. F. B. (2021). Desafios cotidianos e possibilidades de cuidado com crianças e adolescentes com Transtorno do Espectro Autista (TEA) frente à COVID-19. Cadernos Brasileiros de Terapia Ocupacional, 29, e2121. https://doi.org/10.1590/2526-8910.ctoAR2121

\begin{abstract}
$\underline{\text { Resumo }}$
Introduçáo: No cenário atual da COVID-19 e das consequentes medidas emergenciais para seu enfrentamento, é fundamental compreender sobre os impactos gerados por esta condição em grupos sociais que vivenciam condiçóes específicas, como é o caso das crianças e adolescentes com Transtorno do Espectro Autista (TEA). Objetivos: Refletir a partir de aspectos teórico-práticos sobre os desafios e as possíveis implicaçóes da atual pandemia no cotidiano de crianças e adolescentes com TEA, apresentando possibilidades de cuidado fundamentadas na Atenção Psicossocial junto a essa população e suas famílias. Método: Trata-se de um ensaio reflexivo, elaborado com base em açóes desenvolvidas em um projeto de extensão universitária intitulado "Estratégias de cuidado em Saúde Mental Infantojuvenil frente à pandemia da COVID-19”, na Universidade Federal de São Carlos (UFSCar). Resultados: Diferentes estratégias de cuidado frente às implicaçóes da pandemia no cotidiano de crianças e adolescentes com TEA são apresentadas e discutidas, sendo que estas envolveram a organização da rotina, intervençôes informacionais, apoio à família, entre outras. Compreende-se que estas perpassaram o nível individual, assim como consideraram as demandas da família e, de forma mais ampliada, a importância do coletivo e da comunidade. Conclusáo: Evidencia-se a necessidade do investimento em práticas e estudos que se pautem nas particularidades dessa população durante a pandemia, de forma a contribuir para o melhor enfrentamento da situação.
\end{abstract}

Palavras-chave: Transtorno Autístico. Criança. Adolescente. Infecçôes por Coronavírus. Terapia Ocupacional. 


\begin{abstract}
$\underline{\text { Abstract }}$
Introduction: In the current scenario of COVID-19 and its emergency demands, it is essential to understand the pandemic and its possible impacts, especially with regard to the most vulnerable social groups, such as children and adolescents with Autistic Spectrum Disorder (ASD). Objectives: This article aims to reflect on theoretical and clinical aspects of the challenges and possible implications of the current pandemic in everyday lives of children and adolescents with ASD, presenting possibilities of care based on Psychosocial Care for this population and their families. Method: This is a reflective essay, elaborated from the actions developed in a university project entitled "Caring strategies in Child and Adolescent Mental Health in the face of the COVID-19 pandemic", at Federal University of São Carlos (UFSCar). Results: Different caring strategies regarding the implications of the pandemic in the daily lives of children and adolescents with ASD are presented and discussed, and they include the organization of routine, informational interventions, support for the families, among others. It is understood that the caring strategies permeate the individual level and the demands of the family, as well as, more broadly, the importance of the collective and the community. Conclusion: There is a need for further studies related to clinical practices and studies that are based on the particularities of this population during the pandemic, in order to better cope with the situation.
\end{abstract}

Keywords: Autistic Disorder. Child. Adolescent. Coronavirus Infections. Occupational Therapy.

\title{
Introduçáo
}

A COVID-19 é uma doença infecciosa causada pelo novo Coronavírus, identificado pela primeira vez em dezembro de 2019, em Wuhan, na China. A Organização Mundial da Saúde (OMS) declarou, no dia 30 de janeiro de 2020, que se tratava de uma Emergência de Saúde Pública, sendo decretado, em 11 de março, como uma pandemia (Organización Mundial de la Salud, 2020).

A melhor forma de combate à doença tem sido por meio de açóes preventivas, seguindo algumas medidas de controle e proteção, como a lavagem e higienizaçáo das mãos, uso de máscaras e o isolamento social como forma de evitar a propagação da doença (Organização Pan-Americana da Saúde, 2020).

A pandemia da COVID-19 tem gerado uma série de mudanças na vida das famílias e da sociedade de forma geral, podendo impactar não só na saúde física e biológica, mas também na saúde mental. A título de exemplo, o isolamento social e as demais recomendações, necessárias para prevenção e diminuição da transmissão da doença, acabam por modificar de forma significativa o cotidiano, podendo resultar em tensão, medo, estresse e ansiedade (Fundação Oswaldo Cruz, 2020).

Nesse sentido, é preciso refletir sobre como as questôes macrossociais se relacionam com os desdobramentos causados pela doença e com o modo com que ela afeta os diferentes grupos sociais (Farias \& Leite Júnior, 2020). As minorias - indígenas, população em situação de rua, pessoas com deficiências físicas, transtornos mentais, entre outras - configuram-se como grupos sociais que acumulam discriminaçóes e 
consequentemente têm menos acesso aos serviços de saúde e outros direitos sociais, de forma a serem mais impactados em cenários de crise (Kupper \& Heydt, 2019).

Nesse contexto, tem-se as crianças e os adolescentes com Transtorno do Espectro Autista (TEA), que é um transtorno do neurodesenvolvimento, com início na infância, que tem como principais características a presença de dificuldades na linguagem/comunicação, interação social e comportamento (American Psychiatry Association, 2013). Para essa população em específico, pode ser difícil compreender o cenário pandêmico e todas as ramificaçóes decorrentes da COVID-19, principalmente quando se trata das crianças pequenas e/ou aquelas que apresentam deficiências intelectuais e sensoriais concomitantes ao quadro (Houting, 2020).

Assim, as crianças e adolescentes com TEA podem ser considerados mais vulneráveis à COVID- 19, não por serem suscetíveis às complicaçóes do vírus, mas devido às características próprias do quadro clínico que fragilizam a compreensão do cenário pandêmico, assim como as medidas de controle e proteção, expondo-os a maiores riscos de contaminação.

Nessa direçáo, considerando as particularidades presentes nesse quadro, toda a complexidade existente e o cenário pandêmico atual, é fundamental refletir sobre os impactos dessa crise sanitária mundial no cotidiano dessa populaçáo, uma vez que eles naturalmente já vivenciam situaçóes bastante difíceis e desafiadoras.

Com base em uma busca na literatura atual sobre a pandemia da COVID-19 e possíveis associaçóes com o TEA, foram encontradas poucas publicaçóes, sendo em sua maioria produçôes institucionais e informativas que apresentam dicas e orientaçôes às famílias e profissionais quanto ao manejo das dificuldades cotidianas durante a pandemia (Sociedade Brasileira de Pediatria, 2020; Narzisi, 2020; Laboratório de Terapia Ocupacional e Saúde Mental, 2020).

Apenas duas produções advindas de periódicos científicos foram encontradas, em formato de editorial, sendo estas internacionais (Narzisi, 2020; Houting, 2020). Na primeira, o autor afirma que, ainda que necessárias, as medidas de controle e proteção adotadas pelos países para contenção da propagação do vírus e contágio da população precisam ser administradas com cuidado para evitar a intensificação do sofrimento psíquico nas famílias e dificuldades comportamentais nas crianças com TEA. Nessa direção, o texto apresenta dez dicas que visam a ajudar as famílias a lidarem com essas crianças durante a pandemia como explicar às crianças sobre o que é a doença, organizar as atividades cotidianas, ter tempo para atividades lúdicas, uso de jogos para o ensino, terapia online, entre outras (Narzisi, 2020).

Já no editorial proposto por Houting (2020), a autora, que também apresenta TEA, relata sobre o processo do isolamento social e suas implicações na vida de pessoas adultas com esse quadro, considerando que elas têm enfrentado a situação de diferentes formas, a depender dos aspectos pessoais, contextuais, políticos e principalmente dos sistemas de saúde vigentes. Além disso, a autora sinaliza que o momento atual tem mobilizado a comunidade a se engajar em açóes solidárias e a lidarem com as invisibilidades de populaçóes mais vulneráveis.

No Brasil, foram encontrados apenas materiais informativos em formato de manuais e infográficos que abordam sobre a necessidade de uma atenção especial a essa população durante a pandemia (Sociedade Brasileira de Pediatria, 2020; Laboratório de Terapia Ocupacional e Saúde Mental, 2020). 
Frente a esse cenário e demandas emergenciais, considera-se pertinente o desenvolvimento de estudos e práticas pautadas em evidências científicas e que possam favorecer o desenvolvimento de estratégias de enfrentamento da pandemia, principalmente no que se refere a populaçōes específicas, como é o caso da população infantojuvenil com TEA e suas famílias.

Nesse sentido, o presente manuscrito busca refletir sobre os desafios e as implicaçóes da pandemia da COVID-19 no cotidiano de crianças e adolescentes com TEA, e sobre as possibilidades de cuidado com essa população e suas famílias na perspectiva da atenção psicossocial.

Tais reflexôes têm sido elaboradas com base em açôes que se encontram em andamento por meio de um projeto de extensão universitária intitulado "Estratégias de cuidado em Saúde Mental Infantojuvenil frente à pandemia da COVID-19”, na Universidade Federal de São Carlos (UFSCar). O projeto está dividido em quatro frentes de trabalho, sendo que uma delas é voltada às crianças e adolescentes com TEA e suas famílias, por meio da qual algumas ações têm sido realizadas, a saber:

a) Confecção de materiais informativos à comunidade: visando conscientizar a população sobre o que é o TEA e as implicaçôes da pandemia na vida dessas famílias, materiais em formato de infográfico e cartilhas foram elaborados. Estes, além de abordarem sobre a importância das redes de solidariedade e de apoio a essas famílias, ressaltam as particularidades das pessoas com TEA, visando o respeito e a garantia dos seus direitos. Já para as famílias, foram desenvolvidos materiais com o objetivo principal de orientá-las sobre as possíveis estratégias de enfrentamento das dificuldades.

b) Criação de um canal virtual de comunicação e apoio às famílias: As famílias de crianças e adolescentes com TEA, vinculadas a um serviço de terapia ocupacional em município do interior do Estado de Sáo Paulo, foram convidadas a participar de um canal virtual de comunicaçáo coletivo. Nesse canal, sáo abordados diferentes assuntos, baseados em diversas ferramentas. Por exemplo, foi montada uma pasta com dicas de atividades para as crianças no contexto domiciliar; vídeos que abordam sobre rotina e informaçóes relativas a serviços assistenciais foram compartilhados.

c) Proposição de estratégias para a garantia dos direitos das pessoas com TEA: por meio das redes sociais, algumas animaçôes, folders que abordavam sobre os direitos dessa população foram divulgados. Além disso, juntamente com outras instituições do município-alvo das açôes, foi protocolado um pedido na Defensoria Pública quanto à flexibilização do uso de máscaras por essa população, assim como a flexibilização do isolamento social em um possível cenário de lockdown, caso houvesse o agravamento da situação sanitária.

Visando operacionalizar essas açóes, o projeto de extensão conta com uma equipe interprofissional, composta por 17 integrantes, sendo estes docentes, alunos da graduação e pós- graduação em Terapia Ocupacional, Psicologia, Enfermagem e Medicina. Além disso, as açôes são planejadas em encontros virtuais quinzenais sistemáticos com esse grupo. 


\section{O Campo da Saúde Mental Infantojuvenil e a Atençáo Psicossocial: Fundamentos para o Cuidado com Crianças e Adolescentes com TEA}

Nesta seção, pretende-se elucidar brevemente alguns dos principais fundamentos que sustentam as açóes extensionistas desenvolvidas, assim como as reflexóes propostas no presente ensaio. Desta forma, seráo apresentados apontamentos relativos ao referencial teórico-prático da Atenção Psicossocial (Yasui, 2010; Costa-Rosa, 2013) e o que tem sido abordado pelas políticas públicas nacionais que sustentam o campo da saúde mental infantojuvenil (Brasil, 2015).

Costa-Rosa et al. (2003) afirmam que a atenção psicossocial vinculada ao movimento da reforma psiquiátrica sustenta um conjunto de ações teórico-práticas, político-ideológicas e éticas de caráter inovador, com intuito de substituir o modelo asilar. Segundo os autores, não se trata apenas da transformação nos serviços de saúde mental ou de organização institucional, mas, sim, da maneira como se cuida e reinventa a sociedade, dando outro lugar ao sofrimento psíquico, respeitando as diferenças e compreendendo a saúde como produção e promoção da autonomia, liberdade e defesa da vida.

Porém, identifica-se que, quando se trata da saúde mental infantojuvenil, a transformação na assistência se deu tardiamente e muitas vezes atrelada ao cenário dos adultos. Foi somente com a III Conferência Nacional de Saúde Mental, em 2001, que se passou a considerar a construção de políticas públicas e estratégias de cuidado específicas para essa população (Couto \& Delgado, 2015).

Atualmente, a Atenção Psicossocial, no Brasil, apresenta-se como o arranjo estruturante da Rede de Atenção Psicossocial (RAPS), sustentando teórica e metodologicamente o processo de trabalho e de cuidado que orienta seu funcionamento, bem como a organização dos seus dispositivos e práticas. Ainda que a RAPS tenha sido proposta considerando os mais diversos grupos vulneráveis, como a infância e a adolescência, a sua constituição parte daquilo que se tinha enquanto modelo e filosofia de cuidado em saúde mental para a população adulta (Fernandes et al., 2020).

Nesse sentido, considerando as particularidades da infância e adolescência, o Ministério da Saúde lançou, em 2014, um documento intitulado "Atenção Psicossocial a crianças e adolescentes no SUS: tecendo redes para garantir o cuidado”. Este documento aponta para a construção do cuidado em saúde mental infantojuvenil fundamentado em algumas diretrizes e ressalta uma série de aspectos específicos a essa população (Brasil, 2014).

É com base nessa transformação que começa a ser possível vislumbrar um modelo de assistência à infância e à adolescência, bem como uma dinâmica de funcionamento da rede, de forma que pilares de sustentação dessa transformação, como o exercício de direitos de cidadania e a inclusão social, possam realmente ser vivenciados pela população assistida (Fernandes et al., 2020).

Especificamente em relação às crianças e adolescentes com TEA e, apesar das políticas públicas no campo da saúde mental contemplarem algumas de suas particularidades, o Ministério da Saúde publicou, em 2015, a "Linha de Cuidado para a Atençáo às Pessoas com Transtornos do Espectro do Autismo e suas Famílias na Rede de Atenção Psicossocial do Sistema Único de Saúde” (Brasil, 2015), reforçando a compreensão do TEA enquanto um transtorno mental. 
Nesse documento, são apresentados alguns princípios fundamentais relacionados à assistência a essa população, que correspondem à dimensão da Atenção Psicossocial como o estímulo da autonomia, o respeito à subjetividade, a integralidade do cuidado, a garantia dos direitos e da cidadania, a inclusão dos familiares no processo assistência, entre outros (Oliveira et al., 2017).

Reafirmando-se o compromisso de ampliar significativamente o acesso e a qualidade da atenção direcionada às pessoas com TEA e suas famílias, considera-se fundamental que cada ponto de atenção da RAPS se responsabilize em oferecer diversificadas possibilidades de acesso e diferentes modalidades de cuidado para compreender e responder às necessidades das pessoas com TEA em seus contextos de vida (Fernandes et al., 2020). Ou seja, as açôes e estratégias de cuidado devem, além de apostar na construção de autonomia das pessoas com TEA, apoiar sua família para a promoção de sua própria saúde, fomentando e sustentando sua participação nos espaços sociais e coletivos.

Com base nessa perspectiva, apresentam-se, a seguir, algumas consideraçóes referentes às estratégias de cuidado frente às implicaçóes da pandemia no cotidiano de crianças e adolescentes com TEA.

\section{Estratégias de Cuidado Frente às Implicaçóes da Pandemia no Cotidiano de Crianças e Adolescentes com TEA}

Esta seção não possui a pretensão de esgotar as possibilidades e estratégias de cuidado psicossocial junto à população com TEA e suas famílias frente à pandemia da COVID19 e nem de responder a todas as demandas existentes, ainda que o recente cenário pandêmico exija dos profissionais a criação e proposição de ações em tempo real. Compreende-se que os impactos desta doença tanto no cenário mundial quanto no nacional ainda são incalculáveis, por se tratar de uma crise sanitária emergente.

\section{A rotina enquanto elemento protetivo}

A literatura da área sinaliza que a rotina já é um ponto de atenção comumente abordado pelos profissionais que atuam junto a essa população, na medida em que as mudanças que ocorrem no dia a dia possuem grande potencial de produzir sofrimento aos sujeitos com TEA (Brasil, 2015; Machado, 2019). Nessa direção, considerando a sobrecarga emocional e de tarefas que as famílias estão enfrentando, o intuito não é criar novas demandas que poderiam sobrepesar ainda mais o cotidiano destas, mas, sim, amenizar as possíveis dificuldades e sofrimento que possam surgir devido a essa questão.

Acredita-se que organizar a rotina de crianças e adolescentes com TEA, de tal maneira a garantir uma maior previsibilidade das atividades cotidianas, é uma estratégia de cuidado essencial a ser adotada em tempos de pandemia. Essa estruturação pode se dar por meio de diferentes ferramentas, como quadros, imagens/figuras, de forma que eles compreendam o momento de dormir e acordar, fazer as refeiçôes e outras atividades de vida diária, e também pode estar relacionada com a garantia de momentos de lazer e brincadeiras, assim como atividades estruturadas que exigem mais atenção e concentração, como aquelas relacionadas aos conteúdos pedagógicos. Destaca-se, entretanto, que é preciso contextualizar as diferentes realidades e especificidades 
presentes na vida de cada criança e adolescente, assim como as dificuldades já existentes e aquelas que emergiram a partir do cenário atual, de forma a amenizar sentimentos que possam gerar desorganização emocional (Laboratório de Terapia Ocupacional e Saúde Mental, 2020).

Dessa forma, a participação ativa da própria criança/adolescente e de sua família na proposição de tais rotinas é algo a ser fomentado pelos profissionais da saúde mental, visando garantir a subjetividade, tal como é proposto por Costa-Rosa (2000).

\section{Reduzindo os possíveis danos gerados pelo isolamento social às crianças com autismo $\mathrm{e}$ suas famílias}

As medidas de isolamento social e a necessidade de permanecer em casa podem gerar, em alguns momentos, situaçóes de intenso sofrimento - estresse, ansiedade e comportamentos agressivos e autolesivos. Assim, pode ser que as famílias sintam a necessidade de flexibilizar a situaçáo para evitar maiores dificuldades.

Nesse caso, as famílias podem sair de casa com a criança/adolescente, recorrendo, de preferência, a locais abertos e em horários onde há menos movimento nas ruas, para que possam caminhar ao ar livre ou permitir que a criança/adolescente explore ambientes externos à sua casa, considerando toda segurança e buscando garantir a proteçáo necessária.

Ainda que isso não seja o recomendado para a população de forma geral, esta tem sido uma estratégia de cuidado importante tanto para amenizar quanto para prevenir a intensificação do sofrimento das pessoas com TEA, e que tem sido adotada em diferentes países, na forma de legislação, inclusive em contextos nos quais o lockdown foi adotado, como na França e Espanha (Notícias Uol, 2020; Confereración Autismo España, 2020).

Portanto, observa-se que, para além das estratégias de cuidado que envolvem açōes diretas às crianças e adolescentes com TEA e suas famílias, é importante refletir de forma mais ampliada sobre o papel do governo e políticas públicas nesse momento, visando a garantia dos direitos das pessoas com TEA em tempos de pandemia, de modo a amenizar as dificuldades das crianças e adolescentes que vivenciam esta condição e suas famílias frente ao atual cenário.

Considera-se que para a efetiva transiçâo paradigmática do modelo asilar para o psicossocial, existem alguns fatores que podem contribuir para essa transição, e que certamente estão relacionados às singularidades históricas locais, conjunturais e políticas (Mondoni \& Rosa, 2010). É preciso investir e transcender algumas barreiras, tendo em vista a necessidade de ampliação e expansão das políticas e de garantias de execução, considerando, de fato, a complexidade e singularidade da infância e adolescência (Fernandes et al., 2020), como é o caso das crianças e adolescentes com TEA nesse cenário tâo peculiar.

\section{Sobre uso de máscara}

No que se refere às crianças e adolescentes com TEA, em alguns casos o uso de máscara será algo inviável, a depender do nível de comprometimento destes, principalmente para aqueles que apresentam comprometimentos intelectuais e sensoriais. Sendo assim, as tentativas de fazer com que eles a utilizem podem provocar 
intenso sofrimento e crises e, nesse caso, é importante que os demais cuidados e medidas de controle e proteção sejam intensificados (Sociedade Brasileira de Pediatria, 2020).

No dia 02 de julho de 2020, foi sancionada no Brasil a Lei Federal n 14.019, a qual dispensa o uso obrigatório de máscara para pessoas com TEA e outras deficiências que apresentam dificuldades no uso de forma adequada (Brasil, 2020).

Porém, caso o uso de máscara seja uma opção possível de ser adotada, algumas estratégias podem contribuir para que a experiência de uso seja menos desagradável como o uso de tecidos mais maleáveis e texturas suaves para confecção destas, por exemplo, com elásticos mais alargados, ou que sejam presos a algum adereço como arcos ou faixas. Outras estratégias também podem ser adotadas como parte do processo de adaptação e aceitação, como explicar o que é a doença de forma lúdica e, consequentemente, como se prevenir; confeccioná-las de forma lúdica e participativa; inserir a máscara nas brincadeiras, entre outras (Sociedade Brasileira de Pediatria, 2020).

\section{Intervençóes informacionais}

As Intervenções Informacionais são aquelas que buscam a transferência de informaçóes entre as pessoas, e quando utilizadas de forma estratégica podem servir como um elo entre os profissionais da saúde e as comunidades (Moraes, 2008). Intervençôes como estas consideram os diversos contextos culturais, econômicos e sociais das pessoas, de tal maneira a facilitar a transmissão de conhecimento de forma ética e que promova a cidadania, participação social, além de ampliar o debate sobre a diversidade, subjetividade e direitos.

Compreende-se que para avançar na perspectiva da atenção psicossocial, este tipo de estratégia é fundamental, uma vez que abrange diferentes instrumentos utilizados nas intervençóes, possibilidades de intercâmbio entre sujeito, rede de cuidado e comunidade, e considera os fins políticos e socioculturais decorrentes da prática produzida, conforme apresentado por Costa-Rosa (2000).

No cenário atual, faz-se primordial o uso de intervençóes dessa natureza, principalmente com populações mais passíveis de cuidado, como é o caso das crianças e adolescentes com TEA. A título de exemplo, no projeto relatado do presente estudo, a equipe elaborou três materiais em formato de infográfico e uma animação voltados às famílias de crianças e adolescentes com TEA e à comununidade. Nestes, foi possível conscientizar a população sobre o que é o TEA e as implicações da pandemia na vida dessas famílias, abordar sobre a importância e a necessidade de criar redes de solidariedade e de apoio a essas famílias e ressaltar as particularidades das pessoas com TEA, visando o respeito e a garantia dos seus direitos.

Aponta-se que os materiais foram elaborados a partir da identificação da demanda, sendo construídos a partir de uma linguagem acessível, visando o maior alcance. Assim, as produçôes foram divulgadas nos serviços, redes sociais, rádio e televisão.

Nesse sentido, é dever da Universidade, terapeutas e demais atores que trabalham nesse campo e que compóem a rede de cuidados se corresponsabilizarem por ações desta natureza, visto que a rede de cuidados para a infância e adolescência ainda é frágil (Fernandes et al., 2020). 
Considera-se que tanto o conhecimento compartilhado quanto o produzido trazem muitas potencialidades e possibilidades de cuidado para uma população que muitas vezes não tem acesso a essas informaçôes.

\section{A família enquanto alvo da atençáo psicossocial no contexto pandêmico}

Ainda que as estratégias mencionadas nos itens anteriores envolvam diretamente as famílias de crianças e adolescentes com TEA, esta seção busca enfatizar a necessidade do cuidado específico às famílias, uma vez que lidar cotidianamente com as dificuldades advindas do TEA pode estar gerando um sofrimento intenso a quem cuida. Ressalta-se que, na atençáo psicossocial, o objeto do cuidado é a unidade familiar em toda sua complexidade, entendendo-a como integrante fundamental no processo de cuidado (Mielke et al., 2010).

Essa perspectiva encontra respaldo na literatura, que sinaliza sobre a sobrecarga vivenciada por familiares de crianças e adolescentes com TEA, devido ao acúmulo de tarefas destinadas ao cuidado desses indivíduos (Minatel \& Matsukura, 2014). Além disso, apontam que o isolamento social e o sentimento de solidão são frequentes, sendo resultantes das fragilidades das redes de suporte social (Lima \& Couto, 2020). Nesse sentido, é imprescindível oferecer suporte e apoio social no desempenho de tarefas e decisóes para o cuidado com o familiar, buscando fortalecer as relaçôes que podem produzir saúde, considerando que a família faz parte da rede social envolvida nos processos da atenção psicossocial (Coimbra et al., 2005; Pinho et al., 2010).

Em um contexto de pandemia mundial, tais vivências tendem a se intensificar, pois as poucas fontes de apoio no cuidado às crianças e adolescentes foram suspensas, como terapias e escola. Desta forma, estratégias de cuidado que busquem apoiar as particularidades dessas famílias no momento atual se fazem necessárias e urgentes.

Compreende-se que o primeiro passo se refere à conscientização da comunidade sobre as questóes e particularidades que perpassam o universo das pessoas com TEA, principalmente no que tange às demandas atuais advindas da pandemia, para que se tenham uma melhor compreensão dessa realidade e dificuldades e, assim, possibilitar respeito, empatia e solidariedade. Houting (2020) ressalta o quanto o mundo e as pessoas têm se modificado diante dessa nova realidade, o que fica evidente por meio da rede de solidariedade que tem se espalhado em prol das pessoas com TEA e reforça para a importância de continuar garantindo isso para além da pandemia.

Além disso, profissionais da saúde podem viabilizar espaços de escuta e acolhimento para as famílias, sendo um importante recurso para ajudá-las a enfrentar as adversidades do cenário atual. Nesse momento, os espaços podem se concretizar de forma remota, individualmente ou por meio de grupos em aplicativos de mensagens, videoconferências, ligação telefônica etc.

Outra questáo a ser destacada é sobre a perspectiva e receios das famílias sobre o desenvolvimento de seus filhos e continuidade das terapias. Algumas estratégias, denominadas de Telessaúde (Piropo \& Amaral, 2015), podem ser implementadas, entretanto, é importante enfatizar que, em um cenário de maior vulnerabilidade, o acesso a essas tecnologias pode ser limitado, assim como essa modalidade de cuidado tem sido um desafio para os profissionais, usuários e familiares. Como se trata de uma crise sanitária mundial, a cada dia surgem novos desafios e, consequentemente, a 
necessidade de pensar em medidas e estratégias de enfrentamento, em meio a tantas incertezas. Porém, como se observa, nem sempre as especificidades de alguns grupos sociais são abarcadas, como é o caso das crianças e adolescentes com TEA, sendo fundamental tensionar diferentes instâncias e a comunidade para dar visibilidade a essa população.

\section{Consideraçóes Finais}

Com base no panorama apresentado, as reflexōes possibilitaram compreender as particularidades que envolvem as crianças e adolescentes com TEA diante de cenários desafiadores, como é o caso da pandemia. Além disso, com base nas demandas identificadas, estratégias e açōes de cuidado foram abordadas, de forma a abarcar tanto o cuidado às próprias crianças e adolescentes como também às suas famílias. Compreende-se que estas demandas perpassaram a nível individual, considerando as particularidades, assim como consideraram os pedidos da família e, de forma mais ampliada, a importância do coletivo e da comunidade.

Quanto aos limites deste estudo, por se tratar de uma situação nova e, portanto, desafiadora, algumas demandas têm se evidenciado cotidianamente, de forma que náo foi possível responder a todas elas, como as estratégias de cuidado relativas à área da Educação, por exemplo. Assim, sugere-se que estudos futuros se debrucem neste aspecto e em outras necessidades que certamente continuarão a surgir.

\section{Referências}

American Psychiatry Association - APA. (2013). Diagnostic and Statistical Manual of Mental disorders DSM-5. Washington: American Psychiatric Association.

Brasil. (2014). Atenção psicossocial a crianças e adolescentes no SUS: tecendo redes para garantir direitos. Brasília: Ministério da Saúde. Recuperado em 07 de julho de 2020, de

https://bvsms.saude.gov.br/bvs/publicacoes/atencao_psicossocial_criancas_adolescentes_sus.pdf.

Brasil. (2015). Linha de cuidado para a atenção às pessoas com transtornos do espectro do autismo e suas famílias na Rede de Atenção Psicossocial do Sistema Único de Saúde. Brasília: Ministério da Saúde. Recuperado em 08 de junho de 2020 , de

http://bvsms.saude.gov.br/bvs/publicacoes/linha_cuidado_atencao_pessoas_transtorno.pdf

Brasil. (2020, 02 de julho). Lei no 14.019, de 02 de julho de 2020. Altera a Lei no 13.979, de 6 de fevereiro de 2020, para dispor sobre a obrigatoriedade do uso de máscaras de proteçáo individual para circulação em espaços públicos e privados acessíveis ao público, em vias públicas e em transportes públicos, sobre a adoção de medidas de assepsia de locais de acesso público, inclusive transportes públicos, e sobre a disponibilização de produtos saneantes aos usuários durante a vigência das medidas para enfrentamento da emergência de saúde pública de importância internacional decorrente da pandemia da Covid-19. Diário Oficial [da] República Federativa do Brasil, Brasília. Recuperado em 06 de julho de 2020, de http://www.in.gov.br/en/web/dou/-/lei-n-14.019-de-2-de-julho-de-2020-264918074.

Coimbra, V. C. C., Guimarães, J., Silva, M. C. F., Kantorski, L. P., \& Scatena, M. C. M. (2005). Reabilitação psicossocial e família: consideraçôes sobre a reestruturação da assistência psiquiátrica no Brasil. Revista Eletrônica de Enfermagem, 7(1), 99-104. Recuperado em 06 de julho de 2020, de http://www.fen.ufg.br/revista/revista7_1/revisao_01.htm

Confereración Autismo España (2020). El Ministerio de Sanidad dicta una instrucción que permite a las personas con trastorno del espectro del autismo salir a la calle acompañadas mientras dure el estado de alarma. Confereración Autismo España. Recuperado em 29 de maio de 2020, de http://www.autismo.org.es/actualidad/articulo/el-ministerio-de-sanidad-dicta-una-instruccion-que- 
permite-las-personas-con?fbclid=IwAR1yZKNp4QGIBWsOGzDmj8g3Bg4giRkRMGvl5nn2JiMeCTATxxwCPDbF2I

Costa-Rosa, A. (2000). O modo psicossocial: um paradigma das práticas substitutivas ao modo asilar. In P. Amarante (Org.), Ensaios: subjetividade, saúde mental, sociedade (pp. 141-168). Rio de Janeiro: Fiocruz.

Costa-Rosa, A. (2013). Atenção psicossocial além da reforma psiquiátrica: contribuiçōes a uma clínica crítica dos processos de subjetivação na saúde coletiva. São Paulo: Unesp.

Costa-Rosa, A., Luzio, C. A., \& Yasui, S. (2003). Atenção psicossocial: rumo a um novo paradigma na saúde mental coletiva. In P. Amarante, Archivos de saúde mental e atenção psicossocial (pp. 13-44). Rio de Janeiro: Nau.

Couto, M. C. V., \& Delgado, P. G. G. (2015). Crianças e adolescentes na agenda política da saúde mental brasileira: inclusão tardia, desafios atuais. Psicologia Clínica, 27(1), 17-40. https://doi.org/10.1590/0103-5665201500010000

Farias, M. N., \& Leite Júnior, J. D. (2020). Vulnerabilidade social e Covid-19: consideraçóes a partir da terapia ocupacional social. Cadernos Brasileiros de Terapia Ocupacional, Preprint, 1-15. Recuperado em 22 de maio de 2020, de https://preprints.scielo.org/index.php/scielo/preprint/view/494/626

Fernandes, A. D. S. A., Matsukura, T. S., Lussi, I. A. O., Ferigato, S. H., \& Morato, G. G. (2020). Reflexóes sobre a atençáo psicossocial no campo da saúde mental infantojuvenil. Cadernos Brasileiros de Terapia Ocupacional, 28(2), 725-740. https://doi.org/10.4322/2526-8910.ctoarf1870

Fundação Oswaldo Cruz - Fiocruz (2020). Saúde mental e atenção psicossocial na pandemia Covid-19. Recuperado em 08 de junho de 2020, de https://portal.fiocruz.br/sites/portal.fiocruz.br/files/documentos/saude-mental-e-atencao-psicossocialna-pandemia-covid-19-violencia-domestica-e-familiar-na-covid-19.pdf

Houting, J. (2020). Stepping out of isolatin: autistic people and Covid-19. Autism in Adulthood, 2(2), 1-3. https://doi.org/10.1089/aut.2020.29012.jdh

Kupper, H., \& Heydt, P. (2019). The mission billion: access to health services for 1 billion people with disabilities. London School of Hygiene and Tropical Medicine. Recuperado em 22 de maio de 2020, de https://www.lshtm.ac.uk/TheMissingBillion

Laboratório de Terapia Ocupacional e Saúde Mental - LaFollia. (2020). Orientaçōes às famílias de crianças e adolescentes com autismo. Recuperado em 08 de junho de 2020, de https://www.informasus.ufscar.br/wp-content/uploads/2020/04/Orienta\%C3\%A7\%C3\%B5es$\% \mathrm{C} 3 \% \mathrm{~A} 0$ s-fam\%C3\%ADlias-de-crian\%C3\%A7as-e-adolescentes-com-autismo-em-tempos-decoronav\%C3\%ADrus.pdf

Lima, R. C., \& Couto, M. C. V. (2020). Percepçōes sobre o autismo e experiências de sobrecarga no cuidado cotidiano: estudo com familiares de capsi da regiáo metropolitana do Rio de Janeiro. Cadernos Brasileiros de Saúde Mental, 12(31), 217-244. Recuperado em 08 de junho 2020, de https://periodicos.ufsc.br/index.php/cbsm/article/view/69760/43296

Machado, G. D. S. (2019). A importância da rotina para crianças autistas na educação básica. Revista Gepesvida, 1(9), 100-114. Recuperado em 08 de junho de 2020, de http://www.icepsc.com.br/ojs/index.php/gepesvida/article/view/337/171

Mielke, F. B., Kohlrausch, E., Olschowsky, A., \& Schneider, J. F. (2010). A inclusão da família na atenção psicossocial: uma reflexão. Revista Eletrônica de Enfermagem, 12(4), 761-765. http://dx.doi.org/10.5216/ree.v12i4.6812

Minatel, M., \& Matsukura, T. S. (2014). Famílias de crianças e adolescentes com autismo: cotidiano e realidade de cuidados em diferentes etapas do desenvolvimento. Revista de Terapia Ocupacional da Universidade de São Paulo, 25(2), 126-134. https://doi.org/10.11606/issn.2238-6149.v25i2p126-134

Mondoni, D., \& Rosa, A. C. (2010). Reforma psiquiátrica e transição paradigmática no interior do estado de São Paulo. Psicologia: Teoria e Pesquisa, 26(1), 39-47. https://doi.org/10.1590/S010237722010000100006

Moraes, A. F. (2008). Informação estratégica para as açôes de intervenção social na saúde. Ciência \& Saúde Coletiva, 13(Suppl. 2), 2041-2048. https://doi.org/10.1590/S1413-81232008000900008 
Narzisi, A. (2020). Handle the autism spectrum condition during Coronavirus (COVID-19) stay at home period: ten tips for helping parents and caregivers of young Children. Brain Sciences, 10(4), 1-4. https://doi.org/10.3390/brainsci10040207

Notícias Uol (2020). Governo francês flexibiliza regras de confinamento de autistas durante epidemia do coronavírus. Recuperado em 29 de maio de 2020, de https://noticias.uol.com.br/ultimasnoticias/rfi/2020/04/02/governo-frances-flexibiliza-regras-de-confinamento-de-autistas-duranteepidemia-do-coronavirus.htm

Oliveira, B. D. C., Feldman, C., Couto, M. C. V., \& Lima, R. C. (2017). Políticas para o autismo no Brasil: entre a atenção psicossocial e a reabilitação. Physis: Revista de Saúde Coletiva, 27(3), 707-726. https://doi.org/10.1590/s0103-73312017000300017

Organização Pan-Americana de Saúde - OPAS. Organização Mundial da Saúde - OMS. (2020). Folha informativa - Covid-19 (doença causada pelo novo coronavírus) Recuperado em 18 de maio de 2020, de https://www.paho.org/bra/index.php?option=com_content\&view=article\&id=6101:covid19\&Ite $\operatorname{mid}=875$

Organización Mundial de la Salud - OMS. (2020). Brote de enfermedad por coronavirus (COVID-19). Recuperado em 07 de julho de 2020, de https://www.who.int/es/emergencies/diseases/novelcoronavirus-2019

Pinho, L. B., Hernández, A. M. B., \& Kantorski, L. P. (2010). Reforma psiquiátrica, trabalhadores de saúde mental e a "parceria" da família: o discurso do distanciamento. Interface - Comunicação, Saúde, Educação, 14(32), 103-13. https://doi.org/10.1590/S1414-32832010000100009.

Piropo, T. G. N., \& Amaral, H. O. S. (2015). Telessaúde, contextos e implicaçóes no cenário baiano. Saúde em Debate, 39(104), 279-287. Recuperado em 05 de junho de 2020, de https://www.scielo.br/pdf/sdeb/v39n104/0103-1104-sdeb-39-104-00279.pdf

Sociedade Brasileira de Pediatria - SBP. (2020). Nota de Alerta 17 de Abril de 2020 COVID-19e Transtorno do Espectro Autista. Recuperado em 08 de junho de 2020, de https://www.sbp.com.br/fileadmin/user_upload/22455c-NA_-_COVID19_e_Transtorno_do_Espectro_Autista 1_.pdf

Yasui, S. (2010). Rupturas e Encontros: desafios da Reforma Psiquiátrica brasileira. Rio de Janeiro: Editora Fiocruz.

\section{Contribuiçáo das Autores}

Amanda Dourado Souza Akahosi Fernandes, Marina Speranza, Mayara Soler Ramos Mazak e Danieli Amanda Gasparini foram responsáveis pela concepçáo do texto, organização de fontes e análises, redaçáo do texto e revisão. Maria Fernanda Barboza Cid responsável pela concepção, redação e revisão do texto. Todas as autoras aprovaram a versão final do texto.

\section{Autor para correspondência}

Amanda Dourado Souza Akahosi Fernandes e-mail: amanda.d.fernandes@hotmail.com

\section{Editora de seçáo}

Profa. Dra. Ana Paula Serrata Malfitano 